\title{
A new device to present textured stimuli to touch with simultaneous EEG recording
}

\author{
José M. Reales Avilés and Francisco Muñoz Muñoz \\ Universidad Nacional de Educación a Distancia, Madrid, Spain \\ DieTER KLEINBöHL \\ Mannheim University, Mannheim, Germany \\ AND \\ Manuel Sebastián and Soledad Ballesteros Jiménez \\ Universidad Nacional de Educación a Distancia, Madrid, Spain
}

\begin{abstract}
The study of touch has recently grown, due mainly to the extensive use of several types of actuators that stimulate several subsystems of touch. There is a widespread interest in applying these mechanisms to the study of the neurophysiological correlates of tactual perception. In this article, we present a new device (the tactile spinning wheel [TSW]) for delivering textured surfaces to the finger pad. The TSW allows one to control several parameters of the stimulation (angular speed, texture, etc.) and, connected to an EEG recording system, makes it possible to study neural electrophysiological events. The device consists of a rotating platform on which the tactile stimuli are fixed, a system that synchronizes stimuli onset with the EEG system, and an electronic interface that controls the platform. We present the technical details of the TSW, its calibration, and some experimental results we have obtained with this device.
\end{abstract}

In the last decades, there has been a strong interest in the experimental study of touch perception (Grunwald, 2008; Heller \& Ballesteros, 2006; Klatzky \& Lederman, 2003), particularly in neuroimaging. Part of this interest has been focused on how the subsystems of touch can be stimulated, separately or in conjunction, by a set of several actuators. For example, touch mechanoreceptors can be stimulated by the bending motion of pins powered by electric currents (Dresel et al., 2008), by means of pneumatic devices (Golaszewski, Zschiegner, et al., 2002; Overduin \& Servos, 2004; Zappe, Maucher, Meier, $\&$ Scheiber, 2004), and also by vibrotactile devices or micropins (Allerkamp et al., 2007; Golaszewski, Siedentopf, et al., 2002), since all of these mechanisms provide mechanical information to mechanoreceptors. Similarly, the temperature receptors in the skin can be stimulated by thermoelectric heat pumps (Granovsky, Matre, Sokolik, Lorenz, \& Casey, 2005). Moreover, the nociceptive receptors in the skin can be stimulated by electrical currents (Iannetti, Zambreanu, \& Tracey, 2006). In the study of vibrotactile perception, many other approaches based on pneumatically or electromechanically driven vibrotactile stimulators, such as piezoelectric wafers and benders, have been used (Briggs et al., 2004; Tannan, Whitsel, \& Tommerdahl, 2006). All these devices, based on the usage of materials with properties well known for years, have brought invaluable knowledge about the mechanisms of human haptic perception.

Nonetheless, a considerable part of this progress has also been due to the development of new mechanical and electronic devices, as well as the availability of a great variety of new materials with properties suitable for the study of touch. These new devices are so constructed as to deliver several stimulus properties to the haptic perceptual system in a controlled way (e.g., by specifying stimulus onset asynchrony or the interstimulus interval [ISI], by presenting different kinds of stimuli in a row, etc.). Such systems are generally referred to as automated tactile delivery systems. The general arrangement of these devices depends on specific experimental purposes, as well as the nature of the stimulus. Regarding the new materials, they have properties that allow for the stimulation of different subsystems of touch (e.g., among others, electrorheological fluids, Kenaley \& Cutkosky, 1989; the ionic polymer metallic composite, Konyo, Akazawa, Tadokoro, \& Takamori, 2003, and Konyo, Tadokoro, Takamori, \& Oguro, 2000; and shape memory alloys, Velázquez, Pissaloux, Hafez, \& Szewczyk, 2007).

Regarding the study of surface texture perception, several procedures have been developed depending on whether the main interest is to create a real or a virtual movement of the texture on the skin. In the former proce-

J. M. R. Avilés, jmreales@psi.uned.es 
dure, the hand usually is kept static, and the texture sensation is produced by rubbing the surface across the skin. This procedure implies that an external agent is moving the object over the hand, which rests static. Concerning the virtual movement procedures, in many other studies, the aim has been to create illusory motion sensations by delivering a train of pulses to adjacent locations in a very quick temporal sequence (Kirman, 1974; Sherrick \& Rogers, 1966; Summers, Francis, Bowtell, McGlone, \& Clemence, 2009). With this last procedure, it is not possible to evoke a genuine roughness perception because, although there appears to be a relative motion between the skin and the tactile surface, a movement by rubbing is not created (Lamb, 1983; Lederman, 1981; Verrillo, Bolanowski, \& McGlone, 1999). Conversely, the virtual movement procedure creates an illusory sensation of textured surfaces displaced over the skin.

In our lab, we developed a new device and experimental protocol for presenting textured surfaces to a subject's resting index finger pad. Similar procedures have been described previously in the literature. Jiang, Tremblay, and Chapman (1997) used a drum with different portions of dotted surfaces ranging from 2 to $5 \mathrm{~mm}$ as a tactile stimulator. This device rotates on its axis at different speeds ranging from 53 to $105 \mathrm{~mm} / \mathrm{sec}$. The normal force being exerted over the drum surface can additionally be controlled. Essick, James, and McGlone (1999) developed a device designed to present several natural surfaces (e.g., velvet, cotton, or plastic mesh) to the subject's cheek with a mechanical forearm. Two directions of movement on the skin were controlled: ventral-dorsal and upwarddownward. The speed of stimulus motion was $0.5,5$, or $50 \mathrm{~cm} / \mathrm{sec}$. These procedures were proposed so that a real sensation of movement over the skin could be created.

The aforementioned procedures allow one to present surfaces in a controlled manner in physiological experiments involving touch receptors or in purely psychophysical experiments (Bergmann Tiest \& Kappers, 2006, 2007, 2008). There is the opportunity to couple those procedures for neuroimaging or electrophysiological experiments addressing psychophysiological hypotheses on somatosensation, perception, recognition, encoding, and so forth. With this purpose in mind, an fMRI-compatible tactile stimulator, the Helix, has been developed that can deliver several stimulus combinations to the skin by means of the lateral movement of a cylinder (Ingeholm et al., 2006). Another example is the so-called Dodecapus, a pneumatic system whose function is to deliver air puffs on the body surface (Huang \& Sereno, 2007). Similarly, a few other tactile stimulators have been dedicated to magnetoencephalography studies (Hoechstetter, Meinck, Henningsen, Scherg, \& Rupp, 2002; Hoechstetter et al., 2000). With respect to event-related potentials (ERPs), Gillmeister and Eimer (2007) conducted several experiments in which a solenoid attached to the second segment of the left index finger was used. The solenoid drove a metal rod with a blunt conical tip against the finger whenever a current was passed through it. An alternative approach for studying somatosensory processes, using ERPs, is to present the tactile stimuli by rubbing on the skin. In this way, the four mechanoreception systems (the slowly adapting small receptive fields, the slowly adapting large receptive fields, the rapidly adapting small receptive fields, and the rapidly adapting large receptive fields) are activated (Greenspan \& Bolanowski, 1996).

In this article, we present a new mechanical device, designed in the framework of automated tactile delivery systems. The goal is to deliver textured stimuli in the touch modality. A specific requirement for this device is to assess ERPs to tactile surfaces. The tactile stimulator we present here fulfills this requirement, as we describe in the following sections.

\section{Materials and Methods}

The tactile spinning wheel (TSW) stimulator (see Figure 1) presents textured stimuli to the subject's fingertip in a controlled manner (Patent P200801805). This system is specially designed to elicit ERPs to every item presented by the synchronization between the stimulus onset and the trigger delivery to the EEG recording system.

The TSW is composed of two main components: a revolving circular platform and an interface that links the EEG recording system to the TSW. The spinning platform is a horizontal-revolving disk with 16 rectangular sockets placed on the upper side in which the textured elements are fixed onto the platform. Underneath the platform, a set of pins codifies the stimulus position along the platform into a binary code. Each set of pins per position is transiently read by a radial array of photoelectrical sensors just before the current stimulus passes under the fingertip. This code is transiently cached until the stimulus block makes contact with the fingertip. At this moment, the current position code is latched as a trigger to the EEG recording system.

The platform of the TSW cannot be seen by subjects, because three sheets of black methacrylate prevent the sight of it (one of them is placed just in front of the sub-

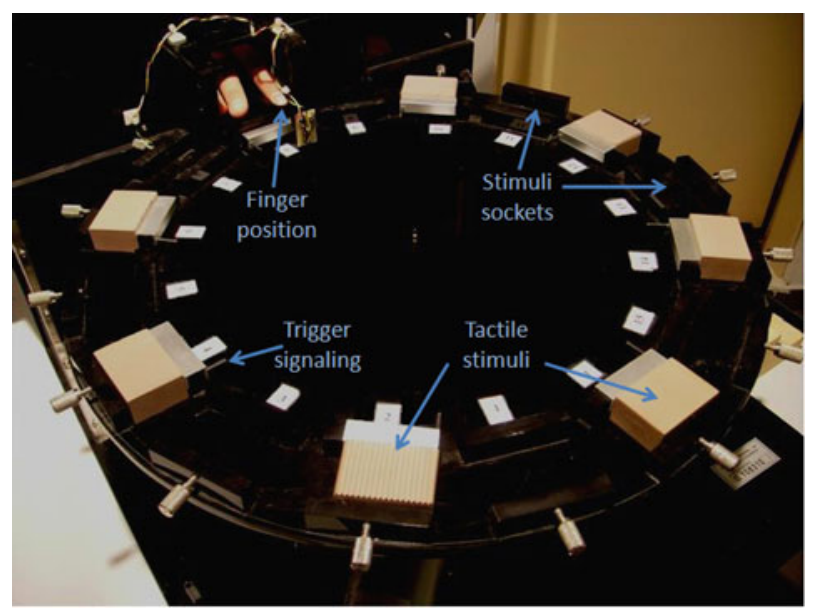

Figure 1. The tactile spinning wheel. The rotating platform is preset with eight stimulus blocks, leaving every second position empty. In the upper left, the index finger is seen, positioned on a perforated tablet allowing the moving stimulus surfaces to rub the fingertip. 
ject, and the other two are placed by the sides of the rotating platform). The engine that powers the platform is located underneath it. An electronic circuitry allows the control of the angular speed of the platform, caches the actual stimulus code, and sends the corresponding digital triggers to the EEG recording device.

In the following section, we will describe the TSW system in more detail concerning the stimulus set, the electronic circuitry, and the mechanical elements.

Stimuli. The set of stimuli used in the present study comprised eight rectangular blocks made of a polymeric compound (polyvinyl). The material was selected according to its property of low thermal conductivity, which rendered the thermal sensation on the skin neutral, avoiding interactions of thermal and tactile qualities of perception. The 3-D dimensions of each prism were $5 \times 3.5 \times 2 \mathrm{~cm}$. Physically, the stimulus blocks differed by the spatial frequency of the triangular gratings embossed on the top side of the blocks, thus modulating the perceptual quality of roughness (Kitada et al., 2005). The embossed grating was produced by carving a triangular profile perpendicularly to the larger dimension of the block with a drill. The groove spacing $(\lambda)$ of the stimulus set we used ranged from 0 to $2.6 \mathrm{~mm}$ in steps of 0.4 per item. The groove depth $(d)$ was approximately $1.8,1.7,1.4,1.1,0.8,0.5,<0.5$, and $0 \mathrm{~mm}$ (see Figure 2).

The stimuli rub the exposed fingertip of the subject at a constant angular speed. Although we used triangular profile elements in our first experiments, it is possible to use different sorts of profiles (e.g., rectangular, sinusoidal, etc.), as well as other physical materials (e.g., metal, plastic, etc.), or to combine many other surface properties (e.g., dot density, length orientation, etc.) as long as the physical dimensions of the elements fit within the sockets and the height does not exceed $2 \mathrm{~cm}$. In fact, the possibility of manipulating two or more physical dimensions of the same item and its particular way of presentation distinguish this device from others. This allows for the possibility of implementing a standard procedure for the

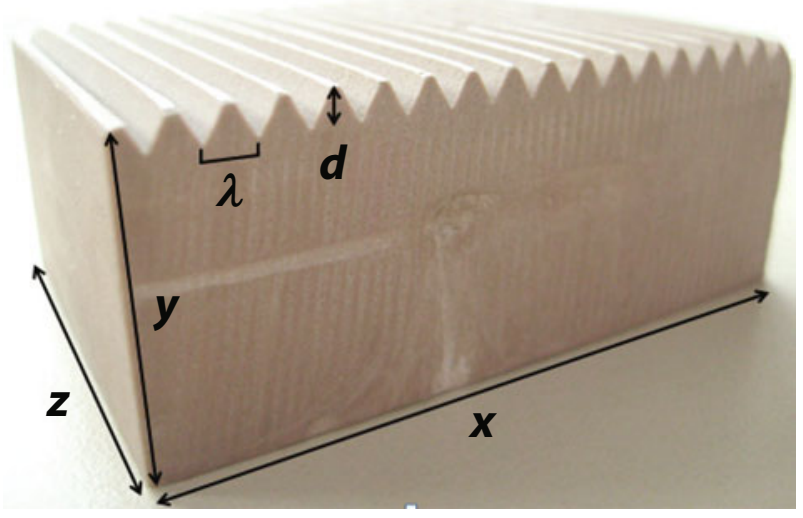

Figure 2. Stimulus block made of polyvinyl. The outer dimensions of the block are $5 \times 3.5 \times 2 \mathrm{~cm}(x \times z \times y)$. A rough triangular grating is engraved at the top, which is the surface moving underneath the fingertip. study of multidimensional properties of tactile surfaces by EEG.

Rotating platform ("spinning wheel"). The rotating platform consists mainly of a revolving mechanical disk used to present textured blocks. The platform is mounted over a vertical axis, which is rotated by a servomotor. Its full radius is $24.6 \mathrm{~cm}$ (corresponding to a perimeter of $154.5 \mathrm{~cm}$ ), and the radius to the center of the finger pad is approximately $20 \mathrm{~cm}$. Both the rotating platform and the stimulus arrangement resemble the original device used by Cholewiak and Collins (1995) in a threshold test of rectangular gratings.

The platform, as well as the main structure of the machine, was built of black methacrylate. The speed of the platform can be directly controlled by a dial placed in the electronic control device. The angular speed of the platform can be adjusted from 0.029 to $0.41 \mathrm{rad} / \mathrm{sec}$.

There are 16 rectangular sockets on the outer circumference of the platform, in which the stimulus blocks are fixed with screws. The inboard dimensions of the sockets are $6.2 \times 3.7 \mathrm{~cm}$. The angle subtended by each textured item in relation to the revolving disk is approximately $13^{\circ}$. The stimulus duration and the ISI can be changed by choosing the number of sockets to be used and by adjusting the speed of the platform. Furthermore, by moving the block in each socket, a degree of jittering of stimulus onset can be introduced to avoid regular expectancies. Placing the stimulus edge at the beginning of the socket, the jittering can be changed in the range of $0-10 \mathrm{~mm}$ forward and 0-20 mm backward.

At the time when the stimulus makes contact with the subject's finger, a photosensor is activated by a pin aligned with the forepart of the stimulus, which releases the electronic trigger to the EEG recording system. Before the delivery of the trigger, the actual stimulus block position has been cached in a buffer with a specific code. Underneath the platform and in the space between two sockets, the code for the incoming stimulus is defined by means of a different distribution of pins aligned radially from the outer part of the platform (see Figure 3).

As was mentioned above, a trigger logic sends a digital signal to the NuAmps amplifier of the EEG recording apparatus. The trigger codes for the actual stimulus item experienced by the subject and marks the stimulus onset for analyzing the ERP (Figure 4). The coding system is a 4-bit code for each position of the spinning platform, allowing discrimination of 16 positions ( $\left.2^{4}\right)$. The Scan Acquire software (Neuroscan) for EEG acquisition can read and display this stimulus code, which is crucial for further offline processing (e.g., epoching and averaging).

The platform revolves at a constant speed by means of a DC servomotor (Model RF-500TB-12560). Speed is kept constant by a feedback control, irrespective of the transversal forces applied to the stimuli. The steady speed control is a key requirement of the system, which avoids slowing down of the platform when the stimulus rubs beneath the finger. Hence, the frictional force being exerted on the stimuli and counteracting the force of the engine can be disregarded. Every revolution of the platform is 


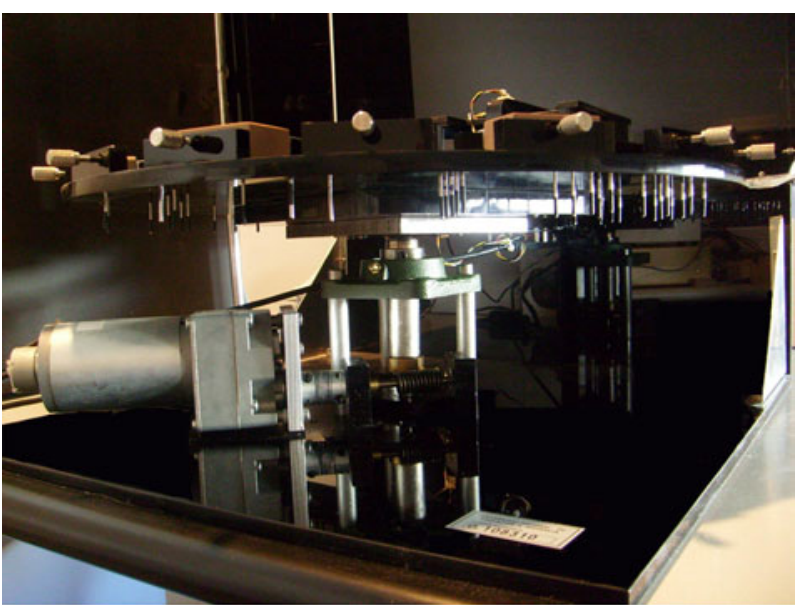

Figure 3. Rotating platform seen from underneath, with the driving motor and gear on the left. The pins coding stimulus positions are seen under each stimulus block.

started and finished manually by a switch placed in the frontal panel of the interface.

The level of noise inside the recording room was recorded by means of a sound level meter (Airflow SLM130). The level of noise was $36 \mathrm{~dB}$ SPL when all equipment was in standby. The TSW interface produced a basal noise of $41 \mathrm{~dB}$ SPL, and when the servomotor was operative, the noise level increased up to $65 \mathrm{~dB}$ SPL in the lower frequency range $(<1 \mathrm{kHz})$. Since the noise of the servomotor was continuous on each run, it was not time locked with the stimulus onset. Therefore, we can disregard any effect on the ERPs.

Controller of the TSW. The electronic core for the control of the DC servomotor is located in the controller module (Figure 5). A negative voltage proportional to the preset angular speed is selected by a potentiometer. This voltage is inverted by unity gain buffers (U1-3) and applied to a Darlington transistor switch (Q1/Q2) capable of switching high currents for starting the DC servomotor (M1). A tachometric dynamo keyed to the servomotor turns in parallel to the servo and provides a positive voltage that is subtracted from the controlling voltage. This circuit operates as a feedback control loop, achieving a constant angular speed when the sum of controlling and feedback voltages is zero.

The TSW is linked with the controller via three separate cable connections: (1) the speed control (DB-15 connector) providing the lines for adjusting the angular speed of the servomotor; (2) the trigger output (DB-25 connector) containing 4-bit lines for the stimulus code; and (3) power for the system.

The trigger interface has an internal logic of 4 bits. When a trigger pulse is delivered from the TSW, the controller holds it in a high state for $100 \mathrm{msec}$. This pulse duration allows appropriate reception of the trigger by external devices - for example, the reprogrammed parallel port of a standard PC (see Figure 6). Optical reflex sensors (OP2 to 5) identify the stimulus code. In the resting state, sensors receive light continuously, thus delivering zero voltage to a D-type flip-flop (MC14175). When the coding pins underneath the platform interrupt the sensor light, the flip-flop circuit latches up the code of the stimulus.

A single pin mounted in line with the edge of the stimulus block on the platform interrupts the light in a photoelectric barrier (OP1), which triggers an electric signal in the comparator U2/1 switching the flip-flop (MC14175). The output from the flip-flop (MC14175) is sent to the digital trigger inputs of the EEG recording system, and a visual cue of the trigger signal is provided to the experimenter by the LED 1.

Calibration in contact force. The distance from the top of each stimulus block to the oval cutout where the fingertip rests has to be precisely constant over all block positions to avoid varying the contact force over stimuli. In order to calibrate the force exerted for every stimulus block on the platform, we used a W00031 force sensor (Entran) connected to an amplifier (Entran, PS-30A) to record the force profile of each stimulus block on the revolving platform. The force exerted on the sensor when the stimulus block passed by was registered on a plotter (Figure 7).

The time course of the exerted force of each stimulus block is nearly identical for all stimulus blocks and can be described as a profile with three phases (Figure 7): stimulus onset, plateau, and offset. The onset phase is the moment at which the stimulus block contacts the sensor. The plateau phase of the force profile shows that the intensity level of the stimuli is nearly the same over all block positions of the spinning wheel platform. The rest period indicates the ISI between each of the stimulus profiles. Small mismatches between the forces exerted by different stimulus blocks were leveled by fixing pieces of a thin foil in the block sockets. So the force exerted by the block on the skin was the same along the whole stimulation set.

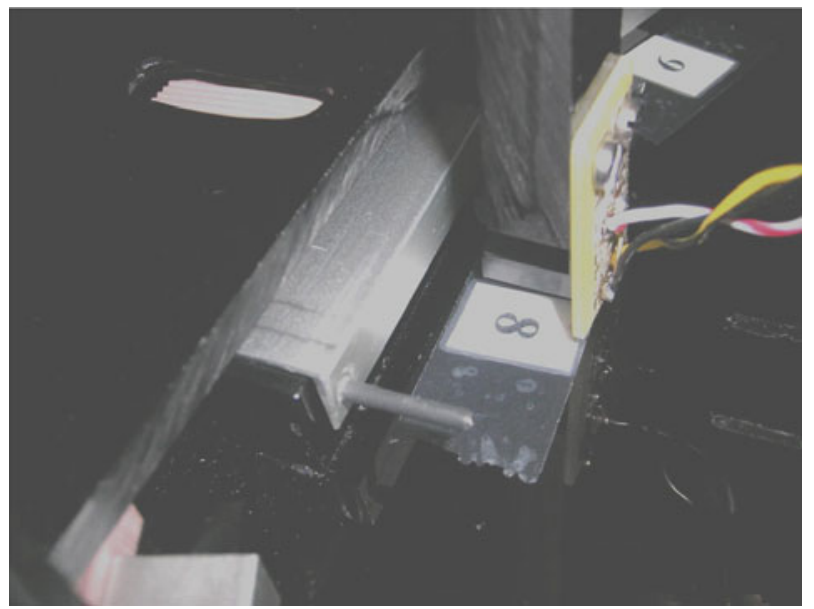

Figure 4. Delivery of a stimulus. The stimulus code has been cached previously; when the trigger pin crosses the photosensor (upper half), the latched code is sent via digital output lines to the EEG recorder. The trigger pin is aligned with the edge of the stimulus item. 


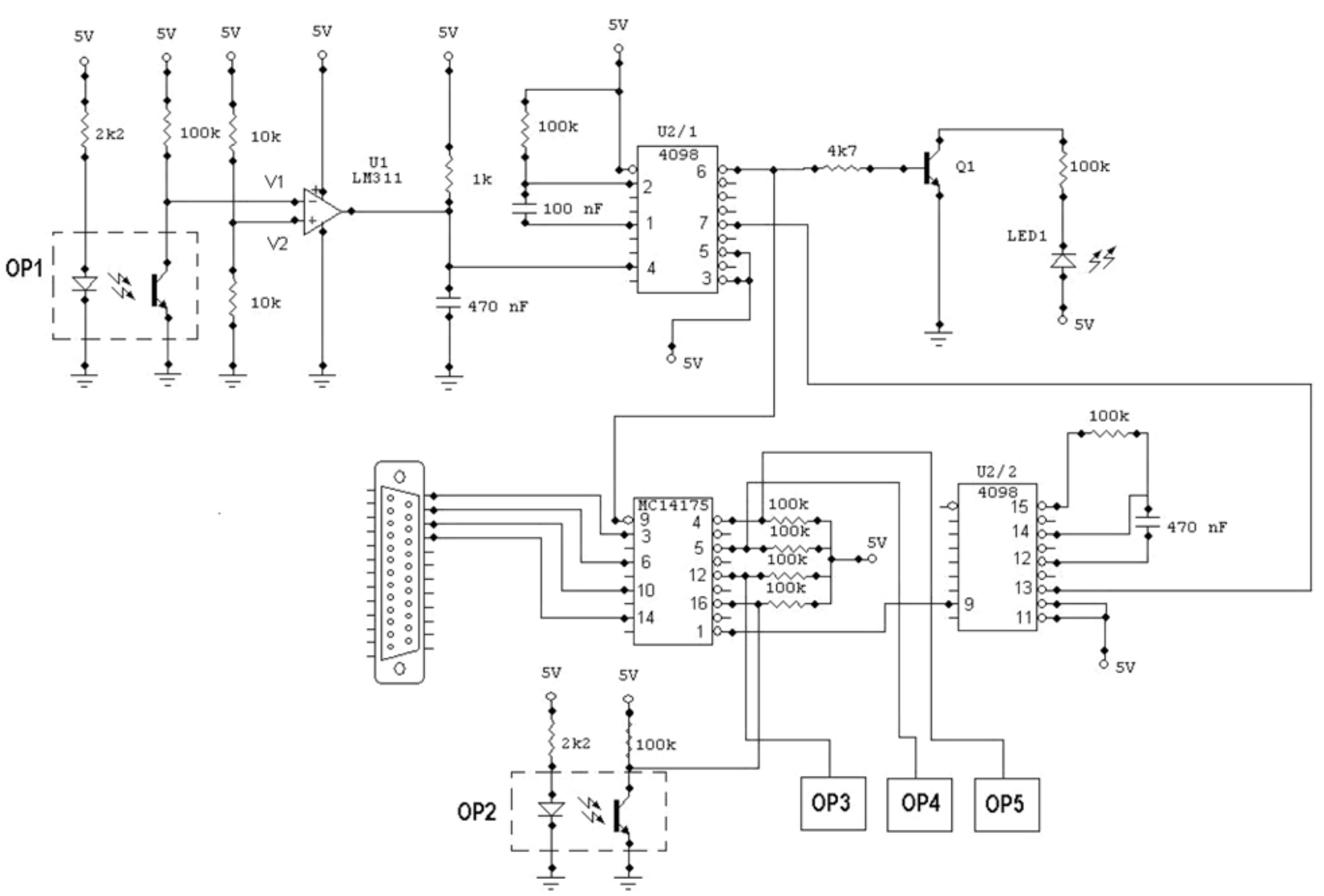

Figure 5. Control circuit for controlling DC servomotor adjustment and feedback control of motor speed (for an explanation, see the text).

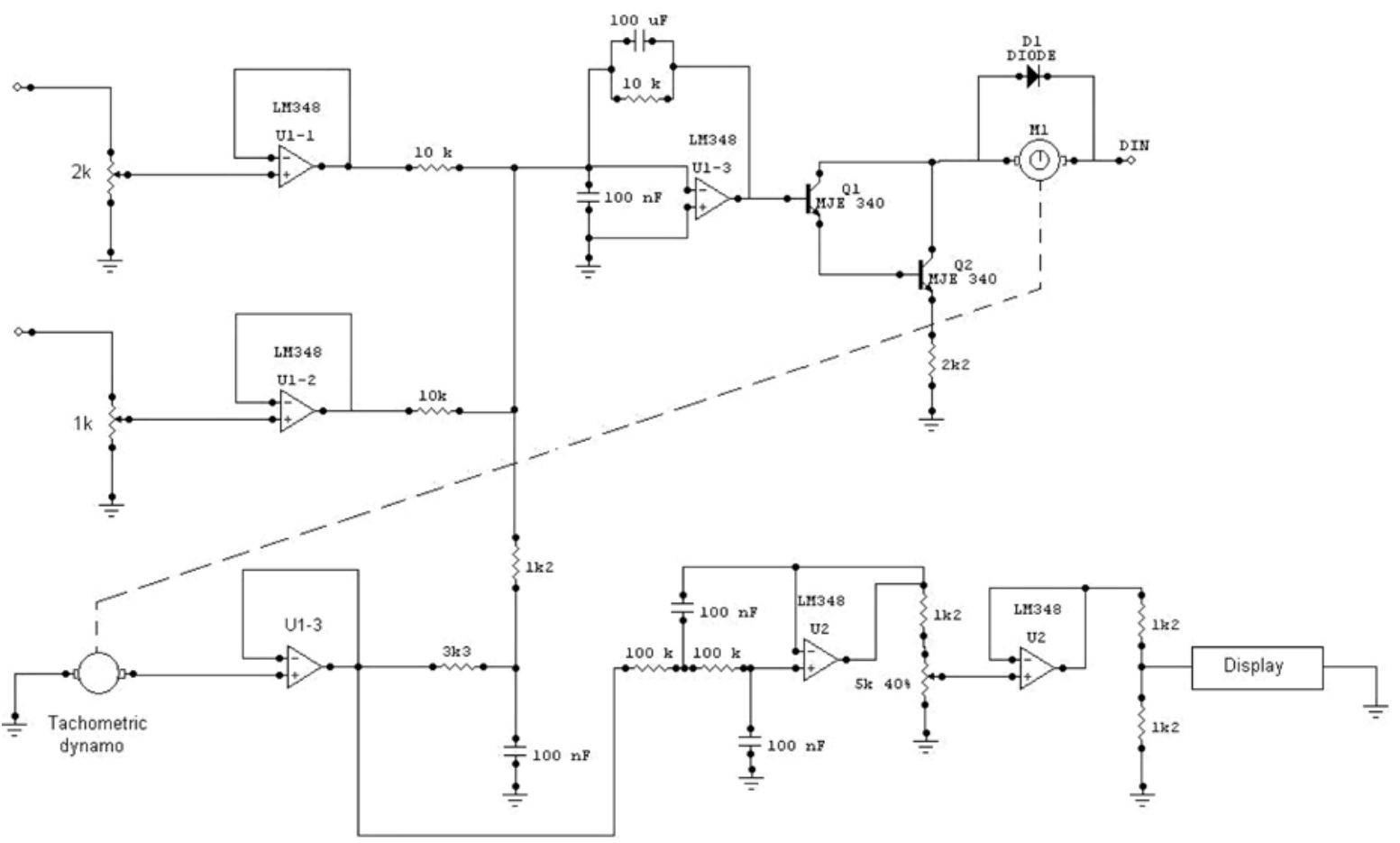

Figure 6. Trigger logic circuit for reading the stimulus block codes and providing the code and a trigger signal synchronized with the first touch of the stimulus edge to the EEG recording equipment (for an explanation, see the text). 


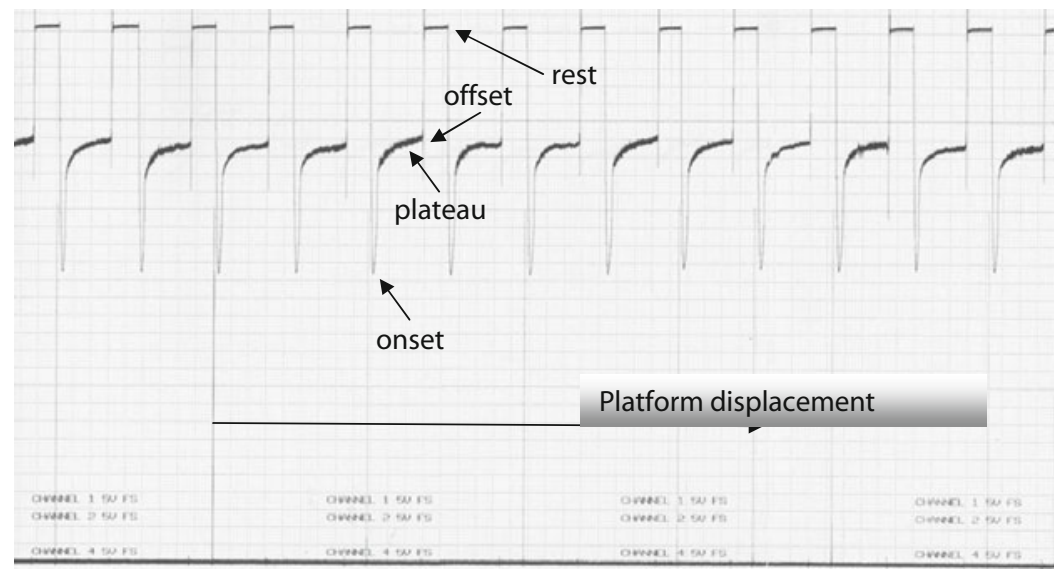

Figure 7. Temporal profile of the force exerted by each stimulus block.

\section{Experimental Testing of the TSW}

The TSW was first tested in an electroencephalographic study using an adapted oddball paradigm. The study investigated tactile discrimination of stimulus blocks with different textured surfaces. The stimulus blocks used in the study consisted of a subset of four textured surfaces: A completely smooth texture was selected, plus three textured stimuli varying in roughness $(0.4,1.6$, and $2.8 \mathrm{~mm}$ of groove width). Three subjects participated in a tactile discrimination task. All the subjects gave their informed consent prior to their inclusion in this test. The study was performed in accordance with the ethical standards laid down in the revised Declaration of Helsinki.

The design used in this test was a blocked design. Each block comprised a randomized sequence of 1 of the 3 textured stimuli (the target) intermixed with instances of the completely smooth texture (the nontarget stimulus, or distractor). In each block, the randomized sequence was divided into trials of 8 stimuli each, corresponding to a complete turn of the TSW. In total, each block consisted of 312 stimuli (39 trials). The proportion of target to nontarget stimuli was $20 / 80$ (20\% target, $80 \%$ nontarget).

In the classical version of the oddball paradigm, the subject is usually asked to count the odd target stimuli. In our study, the subject was asked to count the number of targets presented on each run (one turn of the TSW). At the end of each run, the subject reported the number of targets detected, while the experimenter prepared a new set of stimuli for the next run. Then the spinning wheel moved again, and the subject continued counting from the target count of the last run. At the end of the experiment, the experimenter asked for the total number of targets.

The angular speed of the system was adjusted with the dial on the controller interface. The stimulus duration on the skin of the index fingertip was $560 \mathrm{msec}$, the ISI was $1.78 \mathrm{sec}$, and a complete run took $12.6 \mathrm{sec}$. EEG data were recorded for offline analysis for each subject.

Previous results in other perceptual modalities have shown that attended stimuli elicit larger evoked brain activity than do unattended stimuli (Müller \& Giabbiconi, 2008). The P300 component is a solid index of attentive processes. Our prediction was that the P300 amplitude would be larger for the tactile attended textures, as compared with the unattended textures. We also predicted some kind of P300 modulation as a function of groove width. Finally, we expected that the source of the P300 component would be located at the postcentral cortex.

EEG recordings. The EEG recordings were acquired by means of a 32-channel electrode cap. The electrodes were placed on the scalp according to the International 10-20 system. EEGs were recorded at a sampling rate of $250 \mathrm{~Hz}$ with a NuAmps amplifier, using linked mastoids as reference.

Offline data analysis of EEG was conducted using the EEGLAB software toolbox for MATLAB environment (Delorme \& Makeig, 2004). After filtering the continuous recordings using a digital FIR filter $(0.3-30 \mathrm{~Hz})$, we epoched the EEG recordings in segments of $1,024 \mathrm{msec}$ with a $200-$ msec prestimulus baseline. After visual inspection for muscular and electrode artifacts, an automated Infomax independent component analysis (Makeig, Jung, Bell, Ghahremani, \& Sejnowski, 1997) was performed to subtract ocular artifacts. Artifact-free segments were sorted according to target and nontarget stimuli for averaging. To assess event-related spectral amplitudes for each type of stimulus, a time frequency analysis was performed. This analysis computed the event-related spectral perturbation (ERSP) in the power spectrum for each channel (Makeig, 1993). In an attempt to estimate the 3-D intracerebral current density distribution corresponding to the P300 waveform, we used the LORETA algorithm, a very useful mathematical approach for the computation of topographic images of electric neuronal activity based on extracranial measurements (Pascual-Marqui, Michel, \& Lehmann, 1994).

Results. We obtained evoked brain activity to the four textured surfaces $(0.0,0.4,1.6$, and $2.8 \mathrm{~mm}$ of wavelength). The performance of the subjects in the counting task was nearly perfect. Selective attention engaged in the stimulus evaluation and response selection processes was revealed by a P300-like waveform. Figure 8A shows that a P300-like component was absent for the nontarget unat- 

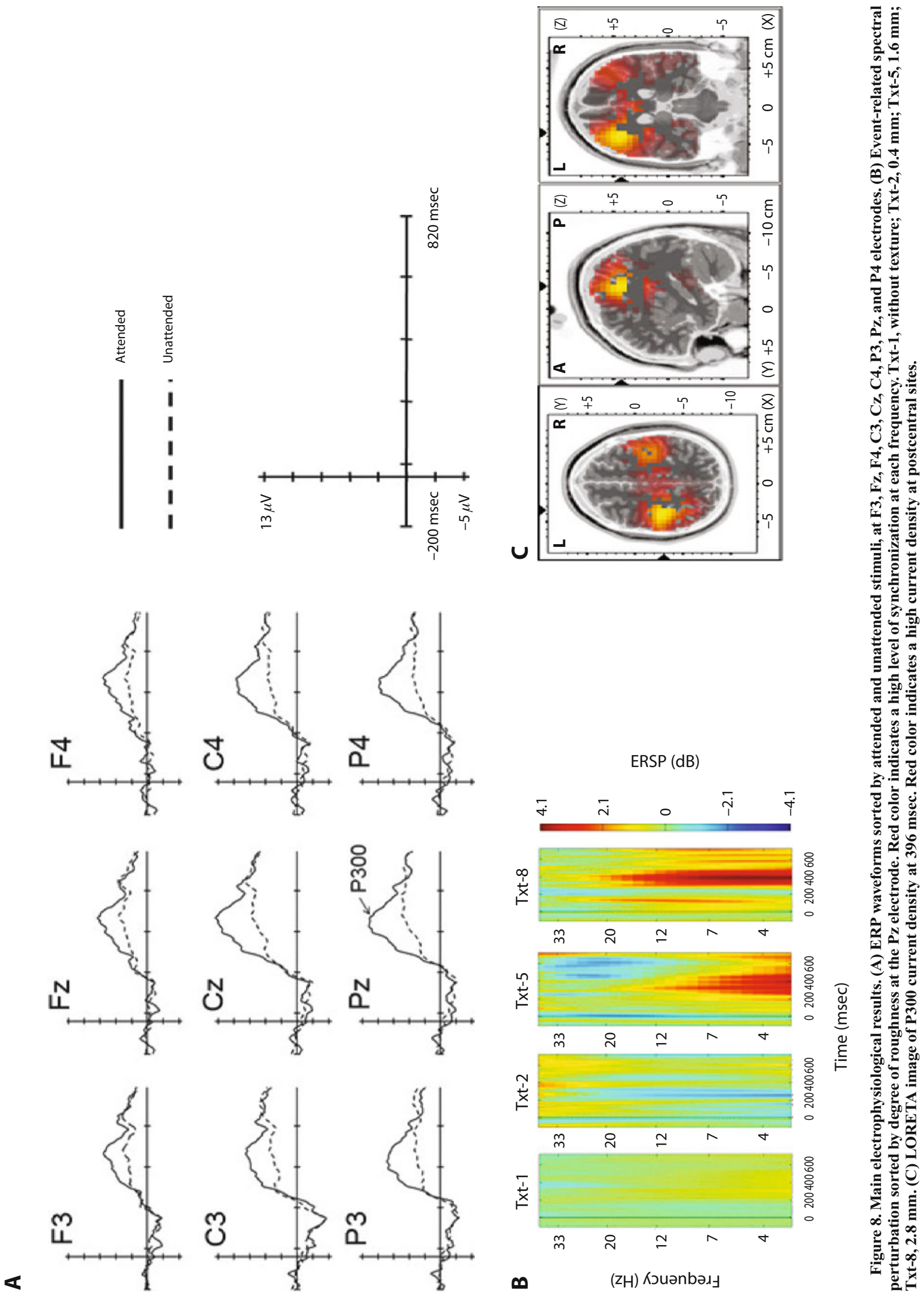
tended texture. By contrast, when all the attended stimuli (the three target textures presented during the experiment) were collapsed, a clear P300 component was obtained.

A time frequency analysis was performed to evaluate the transient synchronization in the slow frequency bands (Figure 8B). Within the theta band, this analysis showed that the highest neural synchronization was achieved for the roughest target stimuli, whereas the lower ERSP amplitude corresponded to the smoothest stimuli. Specifically, the most discriminable stimuli (1.6 and $2.8 \mathrm{~mm})$ showed high spectral power around $400 \mathrm{msec}$ at the $\mathrm{Pz}$ electrode. There was a match with the peak latency of the P300 component. On the contrary, the ERSP was really attenuated with the $0.4-\mathrm{mm}$ roughness stimuli. As was expected, the smooth nontarget stimulus $(0.0 \mathrm{~mm})$ showed the lowest ERSP amplitude, as compared with the other stimuli. The LORETA algorithm showed that the local maximum of the current density distribution (around $400 \mathrm{msec}$ ) was located (see Figure 8C) at the postcentral cortex $\left(0.155 \mu \mathrm{A} / \mathrm{mm}^{2}\right)$. This finding fits nicely with the interpretation according to Bauer, Oostenveld, Peeters, and Fries (2006).

\section{Discussion}

The spinning wheel was designed with the main purpose of presenting textured surfaces to the finger pad in a controlled way, allowing researchers to obtain evoked brain activity for each surface. We achieved this goal by means of a revolving platform with sockets for inserting textured stimuli and with an array of pins for synchronizing the onset of stimulus presentation and the recording of the EEG signal.

Future improvement of the TSW could include measurement of the pressure exerted on the fingertip by the stimuli. To this end, we could monitor this variable so that it could be taken into account in the analysis of ERPs. This monitoring may be achieved by several pressure sensors located below each socket. Another way of monitoring could be to use some psychophysiological measure in the subject's finger related with the pressure. A further line of improving the TSW would be to deliver the stimuli automatically on each run. One way to achieve automatic tactile stimulation could be to continuously move a belt into which several tactile computer-controlled actuators have been inserted. An example of this sort of actuator would be the one described by Velázquez et al. (2007).

To our knowledge, this is the first experiment that has been able to record ERPs for texture surfaces moving under the skin. The results yielded a P300-like component for the target stimulus. The P300 component was similar within those targets with a similar degree of discriminability, independently of their physical attributes. The ERP for the smooth target stimulus $(0.4 \mathrm{~mm})$ was attenuated.

Ballesteros, Muñoz, Sebastián, García, and Reales (2009) used the TSW to investigate the extent to which two tactile textured surfaces varying in roughness modulate early stages of brain activations, as well as whether these brain activations change as a function of the velocity at which the stimulus moves below the fingertip. As was expected, behavioral results showed that both textures were perceived differently, irrespective of the speed at which the stimuli contacted the bare index fingertip of the perceiver. One improvement of the TSW that is additionally being explored at this time is the attachment of several pressure transducers to each stimulus block in order to obtain measurements of the actual applied force concurrently with the EEG recording. In this way, the role of pressure could be taken into account in the EEG analyses.

In conclusion, the TSW allows for a novel way of presenting and recording textured stimuli to be studied by electroencephalographic methods. At present, we are investigating how several combinations of texture attributes influence the EEG.

\section{AUTHOR NOTE}

This work was supported by a grant from the European Community: FP6 NEST-2005-Path-IMP Grant 043432 (SOMAPS). We thank Miguel Angel Sebastián, head of the Department of Construction and Fabrication Engineering (UNED), for the preparation of the tactile stimuli. Correspondence concerning this article should be addressed to J. M. Reales Avilés, Department of Methodology, Universidad Nacional de Educación a Distancia, c/Juan del Rosal, 10, 28040 Madrid, Spain (e-mail: jmreales@psi.uned.es).

\section{REFERENCES}

Allerkamp, D., Böttcher, G., Wolter, F.-E., Brady, A. C., Qu, J., \& SuMmers, I. R. (2007). A vibrotactile approach to tactile rendering. The Visual Computer, 23, 97-108. doi:10.1007/s00371-006-0031-5

Ballesteros, S., Muñoz, F., Sebastián, M., García, B., \& Reales, J. M. (2009). ERP evidence of tactile texture processing: Effects of roughness and movement. In Proceedings of WorldHaptics (WHC'09) (pp. 166-171). Los Alamitos, CA: IEEE Computer Society Press. doi:10.1109/WHC.2009.4810901

Bauer, M., Oostenveld, R., Peeters, M., \& Fries, P. (2006). Tactile spatial attention enhances gamma-band activity in somatosensory cortex and reduces low-frequency activity in parietooccipital areas. Journal of Neuroscience, 26, 490-501. doi:10.1523/ JNEUROSCI.5228-04.2006

Bergmann Tiest, W. M., \& Kappers, A. M. L. (2006). Analysis of haptic perception of materials by multidimensional scaling and physical measurements of roughness and compressibility. Acta Psychologica, 121, 1-20.

Bergmann Tiest, W. M., \& Kappers, A. M. L. (2007). Haptic and visual perception of roughness. Acta Psychologica, 124, 177-189.

Bergmann Tiest, W. M., \& Kappers, A. M. L. (2008). Thermosensory reversal effect quantified. Acta Psychologica, 127, 46-50. doi:10.1016/j.actpsy.2006.12.006

Briggs, R. W., Dy-Liacco, I., Malcolm, M. P., Lee, H., Peck, K. K., GopinATH, K. S., ET AL. (2004). A pneumatic vibrotactile stimulation device for fMRI. Magnetic Resonance in Medicine, 51, 640-643. doi: $10.1002 / \mathrm{mrm} .10732$

Cholewiak, R. W., \& Collins, A. A. (1995). Correlates of vibrotactile pattern processing: Sensory, perceptual, and cognitive factors. In R. Eiler \& K. Ollers (Eds.), Proceedings of the 3rd International Conference on Tactile Aids, Hearing Aids and Cochlear Implants (pp. 86101). Miami: University of Miami, Mailman Center.

Delorme, A., \& Makeig, S. (2004). EEGLAB: An open source toolbox for analysis of single-trial EEG dynamics including independent component analysis. Journal of Neuroscience Methods, 134, 9-21. doi:10.1016/j.jneumeth.2003.10.009

Dresel, C., Parzinger, A., Rimpau, C., Zimmer, C., CeballosBaumanN, A. O., \& Haslinger, B. [A.] (2008). A new device for tactile stimulation during fMRI. NeuroImage, 39, 1094-1103. doi:10.1016/j .neuroimage.2007.09.033

Essick, G. K., James, A., \& McGlone, F. P. (1999). Psychophysical assessment of the affective components of non-painful touch. NeuroReport, 10, 2083-2087.

Gillmeister, H., \& Eimer, M. (2007). Tactile enhancement of audi- 
tory detection and perceived loudness. Brain Research, 1160, 58-68. doi:10.1016/j.brainres.2007.03.041

Golaszewski, S. M., Siedentopf, C. M., Baldauf, E., KoppelstaetTER, F., EISNER, W., Unterrainer, J., ET AL. (2002). Functional magnetic resonance imaging of the human sensorimotor cortex using a novel vibrotactile stimulator. NeuroImage, 17, 421-430. doi:10.1006/ nimg.2002.1195

Golaszewski, S. M., Zschiegner, F., Siedentopf, C. M., Unterrainer, J., Sweeney, R. A., Eisner, W., et AL. (2002). A new pneumatic vibrator for functional magnetic resonance imaging of the human sensorimotor cortex. Neuroscience Letters, 324, 125-128. doi:10.1016/S0304-3940(02)00229-X

Granovsky, Y., Matre, D., Sokolik, A., Lorenz, J., \& Casey, K. L. (2005). Thermoreceptive innervation of human glabrous and hairy skin: A contact heat evoked potential analysis. Pain, 115, 238-247. doi:10.1016/j.pain.2005.02.017

GreensPan, J. D., \& BolanOwSKI, S. J. (1996). The psychophysics of tactile perception and its peripheral physiological basis. In L. Kruger (Ed.), Pain and touch (2nd ed., pp. 25-103). San Diego: Academic Press.

Grunwald, M. (ED.) (2008). Human haptic perception: Basics and applications. Boston: Birkhäuser.

Heller, M. A., \& Ballesteros, S. (Eds.) (2006). Touch and blindness: Psychology and neuroscience. Mahwah, NJ: Erlbaum.

Hoechstetter, K., Meinck, H.-M., Henningsen, P., Scherg, M., \& RupP, A. (2002). Psychogenic sensory loss: Magnetic source imaging reveals normal tactile evoked activity of the human primary and secondary somatosensory cortex. Neuroscience Letters, 323, 137-140. doi:10.1016/S0304-3940(02)00130-1

Hoechstetter, K., Rupp, A., Meinck, H.-M., Weckesser, D., BornFLETH, H., STIPPICH, C., ET AL. (2000). Magnetic source imaging of tactile input shows task-independent attention effects in SII. NeuroReport, 11, 2461-2465.

HuANG, R.-S., \& SERENO, M. I. (2007). Dodecapus: An MR-compatible system for somatosensory stimulation. NeuroImage, 34, 1060-1073. doi:10.1016/j.neuroimage.2006.10.024

Iannetti, G. D., Zambreanu, L., \& Tracey, I. (2006). Similar nociceptive afferents mediate psychophysical and electrophysiological responses to heat stimulation of glabrous and hairy skin in humans. Journal of Physiology, 577, 235-248.

Ingeholm, J. E., Dold, G. R., Pfeffer, L. E., Ide, D., Goldstein, S. R., Johnson, K. O., \& Van Boven, R. W. (2006). The Helix: A multi-modal tactile stimulator for human functional neuroimaging. Journal of Neuroscience Methods, 155, 217-223. doi:10.1016/j .jneumeth.2006.01.018

Jiang, W., Tremblay, F., \& Chapman, C. E. (1997). Neuronal encoding of texture changes in the primary and the secondary somatosensory cortical areas of monkeys during passive texture discrimination. Journal of Neurophysiology, 77, 1656-1662.

Kenaley, G. L., \& CUTKosky, M. R. (1989). Electrorheological fluidbased robotic fingers with tactile sensing. In Proceedings of the 1989 IEEE International Conference on Robotics \& Automation (pp. 132136), Scottsdale, AZ.

KIRMAN, J. H. (1974). Tactile apparent movement: The effects of interstimulus onset interval and stimulus duration. Perception \& Psychophysics, 15, 1-6.

Kitada, R., Hashimoto, T., Kochiyama, T., Kito, T., OKada, T., Matsumura, M., ET AL. (2005). Tactile estimation of the roughness of gratings yields a graded response in the human brain: An fMRI study. NeuroImage, 25, 90-100. doi:10.1016/j.neuroimage.2004.11.026
Klatzky, R., \& Lederman, S. J. (2003). Touch. In I. B. Weiner (Series Ed.) \& A. F. Healy \& R. W. Proctor (Vol. Eds.), Handbook of psychology: Vol. 4. Experimental psychology (pp. 147-176). New York: Wiley.

Konyo, M., Akazawa, K., Tadokoro, S., \& Takamori, T. (2003). Tactile feel display for virtual active touch. In Proceedings of the IEEE/ RSJ International Conference on Intelligent Robots and Systems (pp. 3744-3750). Las Vegas. doi:10.1109/IROS.2003.1249737

Konyo, M., Tadokoro, S., TaKamori, T., \& Oguro, K. (2000). Artificial tactile feel display using soft gel actuators. In Proceedings of the 2000 IEEE Conference on Robotics and Automation (pp. 3416-3421). Los Alamitos, CA: IEEE Computer Society Press.

LAMB, G. D. (1983). Tactile discrimination of textured surfaces: Psychophysical performance measurements in humans. Journal of Physiology, 338, 551-565.

LEDERMAN, S. J. (1981). The perception of surface roughness by active and passive touch. Bulletin of the Psychonomic Society, 18, 253-255.

MAKEIG, S. (1993). Auditory event-related dynamics of the EEG spectrum and effects of exposure to tones. Electroencephalography \& Clinical Neurophysiology, 86, 283-293.

Makeig, S., Jung, T.-P., Bell, A. J., Ghahremani, D., \& Sejnowski, T. J. (1997). Blind separation of auditory event-related brain responses into independent components. Proceedings of the National Academy of Sciences, 94, 10979-10984.

Müller, M. M., \& Giabbiconi, C.-M. (2008). Attention in sense of touch. In M. Grunwald (Ed.), Human haptic perception: Basics and applications (pp. 199-206). Boston: Birkhäuser.

Overduin, S.A., \& SERvos, P. (2004). Distributed digit somatotopy in primary somatosensory cortex. NeuroImage, 23, 462-472. doi:10.1016/j .neuroimage.2004.06.024

Pascual-Marqui, R. D., Michel, C. M., \& Lehmann, D. (1994). Low resolution electromagnetic tomography: A new method for localizing electrical activity in the brain. International Journal of Psychophysiology, 18, 49-65. doi:10.1016/0167-8760(84)90014-X

Sherrick, C. E., \& Rogers, R. (1966). Apparent haptic movement. Perception \& Psychophysics, 1, 175-180.

Summers, I. R., Francis, S. T., Bowtell, R. W., McGlone, F. P., \& Clemence, M. (2009). A functional-magnetic-resonance-imaging investigation of cortical activation from moving vibrotactile stimuli on the fingertip. Journal of the Acoustical Society of America, 125, 1033-1039. doi:10.1121/1.3056399

Tannan, V., Whitsel, B. L., \& Tommerdahl, M. A. (2006). Vibrotactile adaptation enhances spatial localization. Brain Research, 1102, 109-116.

Velázquez, R., Pissaloux, E., Hafez, M., \& SzewczyK, J. (2007). Toward low-cost highly portable tactile displays with shape memory alloys. Applied Bionics \& Biomechanics, 4, 57-70.

Verrillo, R. T., Bolanowski, S. J., \& McGlone, F. P. (1999). Subjective magnitude of tactile roughness. Somatosensory \& Motor Research, 16, 352-360. doi:10.1080/08990229970401

Zappe, A.-C., Maucher, T., Meier, K., \& Scheiber, C. (2004). Evaluation of a pneumatically driven tactile stimulator device for vision substitution during fMRI studies. Magnetic Resonance in Medicine, 51, 828-834. doi:10.1002/mrm.20021

(Manuscript received October 16, 2009; revision accepted for publication December 13, 2009.) 